\title{
Fundamental Measurement of Perceived Length and Perceived Area
}

\author{
Sergio Cesare Masin ${ }^{1}$ \\ ${ }^{1}$ Department General Psychology, University of Padua, Padua, Italy \\ Correspondence: Sergio Cesare Masin, Department General Psychology, University of Padua, 35131 Padua, Italy. \\ Tel: 39-049-827-6670. E-mail: scm@unipd.it
}

Received: May 7, $2012 \quad$ Accepted: May 31, 2012 Online Published: July 16, 2012
doi:10.5539/ijps.v4n3p23
URL: http://dx.doi.org/10.5539/ijps.v4n3p23

\begin{abstract}
Using the method of counting mental units the study explored whether judged lengths and area in right triangles, squares, and disks were consistent with the geometric relationships in these surfaces. Judgments were found to consist with these relationships supporting the idea that they were fundamental measures of perceived extents. Fundamental measures obtained by mental counting differ from corresponding measures obtained by magnitude estimation, rating, and nonmetric scaling. Reasons are suggested as to why these other methods may be biased.
\end{abstract}

Keywords: fundamental measurement, mental counting, magnitude estimation, rating, nonmetric scaling

\section{Introduction}

The fundamental measurement of a value of a physical or mental attribute is the counting of how many constant fundamental units of the attribute must be concatenated to reproduce the value being measured (Campbell, 1920). Units of measurement are fundamental when they cannot be described as a function of other units. In physics, examples are units of physical length, time, or mass. In psychology, examples are units of qualitatively different perceptual attributes such as loudness, brightness, length, etc.

Krantz (1972) suggested applying fundamental measurement to perceived length and area since people would be able to count units of perceived length or area in linear or areal extents, respectively. The following tests support this suggestion. Participants who had made magnitude estimations of length of test lines reported concatenating an image of the standard line along the test lines (Hartley, 1977). This concatenation predicts that response time increases with the length of test lines and as the length of the standard line decreases. Both predictions have been empirically verified (Hartley, 1977, 1981; Reed, Hock, \& Lockhead, 1983). Uhlarik, Pringle, Jordan, and Misceo (1980) found similar results for magnitude estimation of frontal size.

Physical fundamental measurement arguably dates back to Euclid (Michell, 2003; Zudini, 2011) and has been formalized through axioms most notably by Hölder (1901). Reese (1943) applied this axiomatic formalization to psychology (Guilford, 1954, pp. 8-11). Subsequent variants of it are discussed in Falmagne (1985, ch. 2). It has been noted that the significance of these formalizations in psychology has been virtually nil (Cliff, 1992; Estes, 1975; Schönemann, 1994). Before these formalizations are made, one needs to test whether people can properly concatenate a constant mental unit. The present study provided this test for perceived length and area.

Indeed, it is an open question whether mental units of length or area used in direct judgments are constant while they are being concatenated. Another open question is whether this concatenation occurs correctly. Judgments of length or area made in mental units can be fundamental measures if these mental units are constant and correctly concatenated. One way to test this hypothesis is the following.

Pythagoras' theorem applies to the concept of right triangle. Suppose one is looking at a right triangle on a frontal parallel plane with this triangle sufficiently away from the borders of the visual field such that shape distortions are negligible. Pythagoras' theorem applies to this visible triangle since this visible triangle has the geometrical properties that define the concept of a right triangle (Giaquinto, 2007). This consideration means that one can use Pythagoras' theorem as a normative model to test judgments of area or side length of triangles. The idea to use the concept of surface area or Pythagoras' theorem as normative models to study area or length judgment was proposed first by Anderson and Weiss (1971) and Weiss and Gardner (1979), respectively.

If judgments of side length expressed in mental units turn out to be consistent with Pythagoras' theorem, the idea that the mental unit is constant is supported. Analogous reasoning applies to the concept of surface area. 
Let the judgments in mental units of the height, base length, hypotenuse length, and area of a frontal-parallel right triangle be $J_{A}, J_{B}, J_{H}$, and $J_{\text {Area }}$, respectively. If mental units of length and area are constant and properly concatenated and if mental units of length are the same for the legs and the hypotenuse, one has that

$$
J_{H}=\sqrt{J_{A}^{2}+J_{B}^{2}}
$$

and

$$
J_{\text {Area }}=k \cdot J_{A} \cdot J_{B}
$$

with $k$ a constant of proportionality.

The following experiments explored these relations. The participants were instructed to judge length in perceived centimeters (Stevens \& Galanter, 1957). Parenthetically, it may be useful to consider that laymen believe that the method of judging length in perceived centimeters measures physical length in the physical domain. However this method measures perceived length in the phenomenal domain. For example, it allowed determining that the inverted-T illusion consists in a large phenomenal lengthening of the vertical line and in a somewhat smaller phenomenal shortening of the horizontal line (Masin \& Vidotto, 1983). This new finding of a shortening of the horizontal line was robust in that it was later replicated using the method of constant stimuli (Rentmeister-Bryant, Slotnick, \& Parker, 2000).

The following experiments also served to test how the measurement of perceived length and perceived area by mental counting compared with the widely used methods of magnitude estimation, rating, and nonmetric scaling.

\section{Experiment 1}

\subsection{Method}

\subsubsection{Participants}

The participants were 24 university students. They were divided into three equally numerous groups, Groups 1, 2, and 3 .

\subsubsection{Test and Standard Stimuli}

Test stimuli were black right triangles with one leg horizontal and the hypotenuse slanting down to the right. The values of height and base length were 1,5,9,13, or $17 \mathrm{~cm}$. Each test stimulus was presented continuously in the middle of a white frontal-parallel $37.5 \times 28.5 \mathrm{~cm}$ monitor screen (Philips Brilliance 190B) until the respective trial terminated. Viewing distance was $165 \mathrm{~cm}$. The illumination level was $150 \mathrm{~lx}$. The set of 25 test stimuli with different combinations of leg lengths may be called the basic set. Table 1 lists these lengths for the basic set.

The sets of test stimuli formed by 9 and by 3 copies of the basic set may be called the Sets A and B, respectively. For each stimulus, Set A was used to obtain three judgments of length of each leg and of the hypotenuse and Set B to obtain three judgments of area. The resulting 300 test stimuli were presented in randomly intermixed order.

The stimuli of Set A were presented at the onset of the corresponding trial. The stimuli of Set B were presented $1.5 \mathrm{~s}$ after the offset of one standard stimulus, or of two simultaneous standard stimuli, presented centrally for $1.5 \mathrm{~s}$.

The single standard stimulus was used for Groups 1 and 2: a red disk with diameter of $5 \mathrm{~cm}$. The two standard stimuli were used for Group 3: two horizontally-aligned horizontally-based red isosceles right triangles separated by a gap of $7 \mathrm{~cm}$, with legs of 0.2 and of $20 \mathrm{~cm}$, hypotenuse slanting down to the right, and smaller standard on the left.

The monitor screen was set on a table at $55 \mathrm{~cm}$ from the table border closest to the participant. A horizontal $40-\mathrm{cm}$ measuring tape with markings for centimeters and millimeters was stuck on this border for viewing by the participant.

\subsubsection{Procedure}

For each test stimulus a 12-point letter in the upper left corner of the screen indicated whether to judge height, base length, hypotenuse length, or area.

Groups 1-3 were instructed to judge length in perceived centimeters and fractions thereof. They were invited to refer to the measuring tape stuck on the border of the table in front of them.

Groups 1-3 were asked to judge area as follows. 
Group 1 (mental counting) was asked to count how many times the area of the smaller of the test and standard stimuli could be contained in the area of the other including possible fractions. This number was taken as the response when the standard stimulus was judged to be equal or smaller than the test stimulus, and the reciprocal of this number was taken as the response when the test stimulus was judged equal or smaller than the standard stimulus.

Group 2 (magnitude estimation) was asked to assign a number to the area of the test stimulus given that the area of the standard stimulus was 100 with the examples of a stimulus area 3 times larger or 3 times smaller than that of the standard stimulus.

Group 3 (rating) was asked to rate the area of the test stimulus with the areas of the smaller and larger standard stimuli being 1 and 100, respectively.

\section{Results for Length}

Table 1 presents the results. The three middle columns report mean judged height, base length, and hypotenuse length. The three columns on the right report mean counted numbers of units, magnitude estimates, and ratings of area from Groups 1-3, respectively.

Table 1. Dimensions of test stimuli and mean judgments of length and area from Experiment 1

\begin{tabular}{|c|c|c|c|c|c|c|c|c|}
\hline \multirow[b]{2}{*}{ Stimulus } & \multicolumn{2}{|c|}{ Leg length $(\mathrm{cm})$} & \multicolumn{3}{|c|}{ Mean judged length } & \multicolumn{3}{|c|}{ Mean judged area } \\
\hline & Height & Base & Height & Base & Hypot. & Group 1 & Group 2 & Group 3 \\
\hline 1 & 1 & 1 & 1.1 & 1.2 & 1.6 & .12 & 12.2 & 3.9 \\
\hline 2 & 1 & 5 & 1.1 & 5.3 & 5.6 & .25 & 28.3 & 6.9 \\
\hline 3 & 1 & 9 & 1.2 & 8.8 & 8.9 & .33 & 37.0 & 9.5 \\
\hline 4 & 1 & 13 & 1.1 & 12.9 & 12.6 & .41 & 35.8 & 10.5 \\
\hline 5 & 1 & 17 & 1.2 & 16.4 & 17.0 & .42 & 49.2 & 11.6 \\
\hline 6 & 5 & 1 & 5.3 & 1.1 & 5.6 & .31 & 26.9 & 6.8 \\
\hline 7 & 5 & 5 & 5.3 & 5.2 & 6.9 & .86 & 76.7 & 15.9 \\
\hline 8 & 5 & 9 & 5.6 & 8.7 & 9.6 & 1.37 & 113.8 & 18.2 \\
\hline 9 & 5 & 13 & 5.4 & 12.0 & 12.5 & 2.03 & 158.8 & 21.2 \\
\hline 10 & 5 & 17 & 5.4 & 16.0 & 15.5 & 2.17 & 199.0 & 23.4 \\
\hline 11 & 9 & 1 & 9.3 & 1.1 & 9.3 & .36 & 35.4 & 8.8 \\
\hline 12 & 9 & 5 & 8.7 & 5.4 & 10.0 & 1.40 & 110.8 & 19.1 \\
\hline 13 & 9 & 9 & 9.0 & 9.2 & 11.6 & 2.01 & 164.2 & 24.2 \\
\hline 14 & 9 & 13 & 9.1 & 12.5 & 14.2 & 2.75 & 225.0 & 31.0 \\
\hline 15 & 9 & 17 & 8.9 & 15.6 & 16.3 & 3.53 & 306.3 & 32.2 \\
\hline 16 & 13 & 1 & 13.4 & 1.1 & 13.2 & .37 & 42.3 & 10.4 \\
\hline 17 & 13 & 5 & 12.7 & 5.4 & 13.6 & 1.90 & 157.5 & 22.2 \\
\hline 18 & 13 & 9 & 12.8 & 8.9 & 14.6 & 3.15 & 231.7 & 28.3 \\
\hline 19 & 13 & 13 & 12.8 & 12.6 & 16.3 & 4.23 & 340.8 & 38.2 \\
\hline 20 & 13 & 17 & 12.6 & 16.6 & 18.1 & 5.02 & 375.8 & 46.0 \\
\hline 21 & 17 & 1 & 17.4 & 1.1 & 17.3 & .46 & 54.8 & 12.6 \\
\hline 22 & 17 & 5 & 17.0 & 5.2 & 17.2 & 2.09 & 194.6 & 24.0 \\
\hline 23 & 17 & 9 & 16.2 & 8.9 & 17.7 & 3.77 & 301.7 & 34.0 \\
\hline 24 & 17 & 13 & 16.1 & 12.4 & 19.1 & 4.96 & 406.3 & 50.3 \\
\hline 25 & 17 & 17 & 16.6 & 16.3 & 20.2 & 6.67 & 510.4 & 62.0 \\
\hline
\end{tabular}

\subsection{Legs}

The following analyses support the hypothesis that participants judged height $\left(J_{A}\right)$ and base length $\left(J_{B}\right)$ using virtually the same mental unit. A 2 (orientation) $\times 5$ (judged leg) $\times 5$ (non-judged leg) analysis of variance showed the effect of orientation was significant with that of non-judged leg not significant, $F(1,23)=4.7, p<.05$, and $F(4,92)=2.1$, respectively. The effect of orientation occurred essentially only for long and thin stimuli. With stimuli with the shortest leg excluded from analysis, all factors except judged leg and all interactions were not significant, $F(1,23)=3.3, F(3,69)=0.4$ or $2.1, F(4,92)=1.5$, and $F(12,276)=1.6$ or 1.7 .

In Figure 1, to visually emphasize the negligible effect of orientation, mean judged leg length was plotted against physical length. The line shows a least-squares fit to a mean straight line with slope not significantly different 
from 1 and positive intercept significantly different from $0, t(23)=1.35$ and $t(23)=4.11, p<.001$, respectively. The quadratic trend of judged leg length was not significant, $F(1,23)=2.4$.

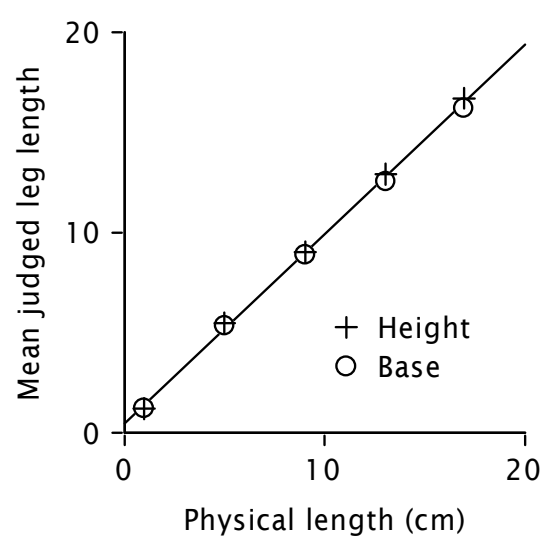

Figure 1. Mean judged height and base length of right triangles plotted against physical length

\subsection{Hypotenuse}

The following analysis shows that the mental unit for the hypotenuse was not constant. In each of 10 pairs of stimuli, hypotenuse length was the same and the longer leg was vertical in one stimulus and horizontal in the other. A 2 (orientation) $\times 10$ (hypotenuse) analysis of variance showed that orientation and the interaction were significant, $F(1,23)=26.9, p<.001$, and $F(9,207)=2.8, p<.005$, respectively. These results agree with those of Weiss and Gardner (1979) and with the finding that produced line length varies with line inclination (Hartley, 1977).

\subsection{Pythagoras' Theorem}

Figure 2, left diagram, shows mean judged hypotenuse length $\left(J_{H}\right)$ plotted against mean perceived hypotenuse length calculated by Equation 1. With mental units constant and properly concatenated, Equation 1 predicts that data points lie on the straight line with slope 1 and intercept 0 . Obtained data points progressively deviated from this line as hypotenuse length increased, RMSD $=1.13$.

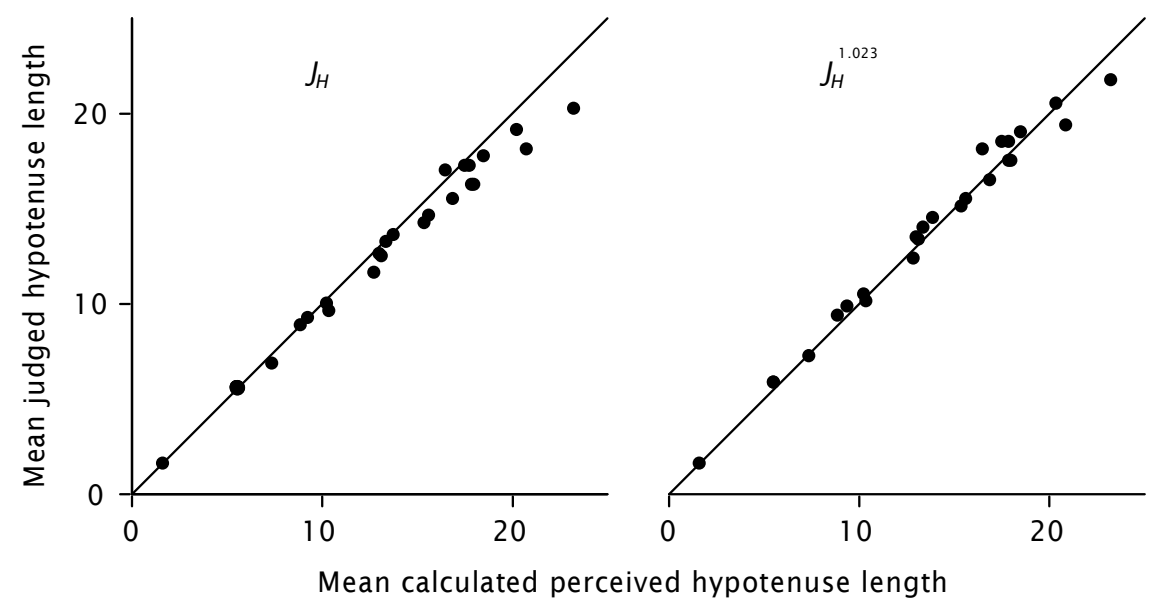

Figure 2. Mean judged hypotenuse length (left) and mean judged hypotenuse length raised to the power of 1.023 (right) plotted against mean calculated perceived hypotenuse length

This deviation may be due to the nonconstancy of the mental length unit for the hypotenuse. This nonconstancy can be compensated by transforming judged hypotenuse length from $J_{H}$ to $J_{H}{ }^{q}$. In Figure 2 the right diagram shows mean $J_{H}^{q}$ with $q=1.023$ plotted against mean calculated perceived hypotenuse length yielding the minimum RMSD of 0.68 . Transformed data points lie fairly well on the straight line with slope 1 and intercept 0 . 


\subsection{Conclusion}

The results support the possibility that $J_{A}$ and $J_{B}$ were fundamental measures of perceived length expressed in virtually the same constant mental unit with $J_{H}$ expressed in a nonconstant mental unit. If $J_{A}$ and $J_{B}$ are fundamental measures then the transformed judgments of hypotenuse length, $J_{H}{ }^{q}$, also are fundamental measures.

\section{Results for Area}

Figure 3 shows mean counted numbers of units, magnitude estimates, and ratings of area plotted against mean perceived area calculated by the expression $J_{A} \cdot J_{B} / 2$. The curves show least squares fits to a standard power function with exponent $0.91,0.84$, or 0.68 .

The near-unity exponent for the method of counting mental units indicates a nearly constant mental unit of area.

For judged area of the 10 pairs of stimuli considered above, orientation and the interaction were not significant for Groups $1-3, F(1,7)=0.5$ and $F(9,63)=0.5, F(1,7)=0.3$ and $F(9,63)=0.3$, and $F(1,7)=0.2$ and $F(9,63)=$ 0.5 , respectively.

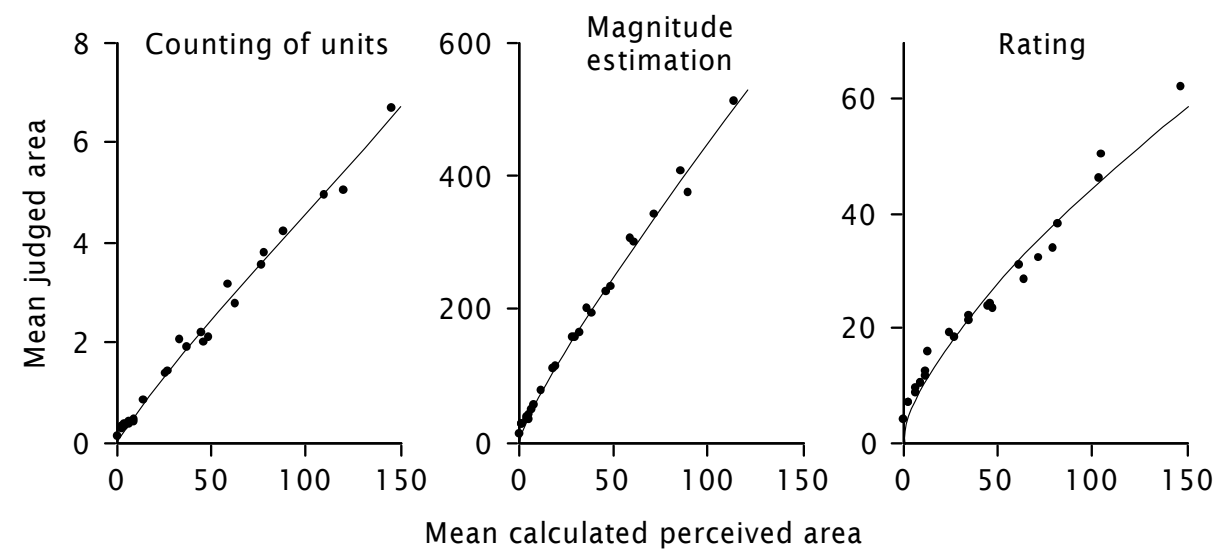

Figure 3. For different judgment methods, mean judged area plotted against mean calculated perceived area of right triangles

\section{Experiment 2}

The curvature in the data in Figure 3 may depend on the shape transformations required to mentally concatenate a disk in triangles or triangles in a disk. This hypothesis was tested using a square as the standard stimulus for judging the area of squares and disks. It was assumed that it was easier to concatenate a square in a square or in a disk rather than for example a disk in a triangle.

\subsection{Participants}

Ten university students participated in each of two sessions, Sessions 1 and 2. Session 2 took place few minutes after Session 1.

\subsection{Method for Session 1}

\subsubsection{Stimuli}

Stimulus presentation and viewing conditions were identical to those used for Experiment 1. Each test stimulus was a horizontally-based black square or was a black disk presented continuously in the middle of the screen until the respective trial terminated. Table 2 lists the sides and diameters used for the stimuli.

The standard stimulus was a horizontally-based red square with side of $4.5 \mathrm{~cm}$. Its left and bottom sides were at $0.4 \mathrm{~cm}$ from the respective left and bottom sides of the screen. For each side and each diameter of test stimuli, the standard stimulus either appeared $3 \mathrm{~s}$ before the test stimulus for $1.5 \mathrm{~s}$ with interstimulus interval of $1.5 \mathrm{~s}$ or appeared $1.5 \mathrm{~s}$ before the test stimulus remaining visible until the trial terminated. (Test stimuli used in Session 1 were used in Session 2 without the standard stimuli. In Session 1, the successive and simultaneous presentations of standard stimuli served to test whether the perceptual presence of the standard stimuli significantly influenced the participants' judgments. The statistical analysis reported below showed that it did not.) There were a total of 32 trials. The set of these 32 trials was presented 5 times consecutively with trials in random order. 


\subsubsection{Procedure}

The participants were asked to report the number of times the smaller stimulus surface could be contained in the larger one, including possible fractions.

\subsection{Method for Session 2}

\subsubsection{Stimuli}

Stimuli, presentation conditions, viewing conditions, and number of randomly presented trials were identical to those used in Session 1. No standard stimulus was used.

\subsubsection{Procedure}

The participants were asked to judge the lengths of side and perimeter of stimulus squares and of diameter and circumference of stimulus disks in perceived centimeters and fractions thereof. Hereafter these lengths are called side, perimeter, diameter, and circumference, respectively. On the upper left corner of the monitor screen a 12-point letter indicated for each stimulus which length to judge.

\section{Results and Discussion}

Table 2 reports mean judgments of side, perimeter, diameter, circumference, and square and disk area. A 2 (shape) $\times 2$ (mode of presentation of standard) $\times 8$ (area) ANOVA showed that all factors except area and all interactions were not significant, $F_{\mathrm{s}}(1,9)=.09$ to 2.9 and $F \mathrm{~s}(7,63)=1.0$ to 1.7 .

Table 2. Dimensions of test stimuli and mean judgments of length and area from Experiment 2

\begin{tabular}{|c|c|c|c|c|c|c|c|}
\hline \multicolumn{2}{|c|}{ Physical length (cm) } & \multicolumn{4}{|c|}{ Mean judged length } & \multicolumn{2}{|c|}{ Mean judged area } \\
\hline Side & Diameter & Side & Perimeter & Diameter & Circumference & Square & Disk \\
\hline 1.5 & 1.69 & 1.49 & 5.72 & 1.69 & 4.44 & 0.13 & 0.14 \\
\hline 3.0 & 3.39 & 2.86 & 10.00 & 3.44 & 9.98 & 0.48 & 0.55 \\
\hline 4.5 & 5.08 & 4.24 & 15.49 & 4.73 & 14.40 & 0.98 & 0.88 \\
\hline 6.0 & 6.77 & 5.43 & 21.14 & 6.20 & 17.62 & 1.53 & 1.43 \\
\hline 7.5 & 8.46 & 6.71 & 25.60 & 7.43 & 19.66 & 2.50 & 2.10 \\
\hline 9.0 & 10.15 & 8.36 & 30.44 & 9.26 & 23.40 & 3.64 & 3.16 \\
\hline 10.5 & 11.85 & 9.67 & 35.95 & 10.27 & 28.00 & 4.85 & 4.71 \\
\hline 12.0 & 13.54 & 10.81 & 41.58 & 12.34 & 31.74 & 6.20 & 6.07 \\
\hline
\end{tabular}

\subsection{Psychophysical Functions}

Figure 4 shows mean judgments of side and diameter and of square and disk area plotted against physical length and physical area, respectively. In each diagram a curve represents a least-squares fit to a mean standard power function with additive offset and with exponent of 1 .
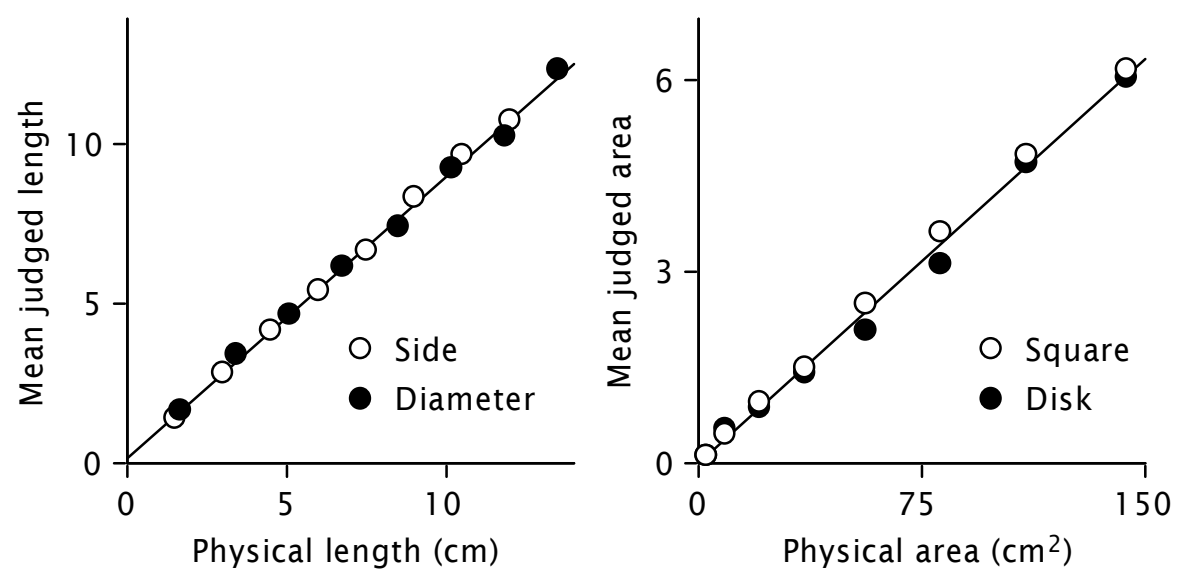

Figure 4. Mean judged lengths of side and diameter and mean judged areas of square and disk plotted against physical length and area, respectively 


\subsection{Calculated Perimeter and Circumference}

Figure 5 shows mean judged perimeter and circumference plotted against mean perceived length calculated by the expressions $4 \cdot J_{L}$ and $\pi \cdot J_{D}$, with $J_{L}$ and $J_{D}$ being the mean judged side and diameter, respectively. Two lines represent the straight line with unit slope and zero intercept. The results support the hypothesis that mental units of length were nearly constant for relatively short perimeters and circumferences, progressively increasing with perimeter or circumference for relatively long lengths.

By magnitude estimation with no designated standard and no assigned modulus, Teghtsoonian and Teghtsoonian (1971) had participants judge diameter and circumference of circles. The judged circumference was less than the circumference calculated from the respective judged diameter, but in this case it is very possible that participants used different moduli for diameter and circumference.

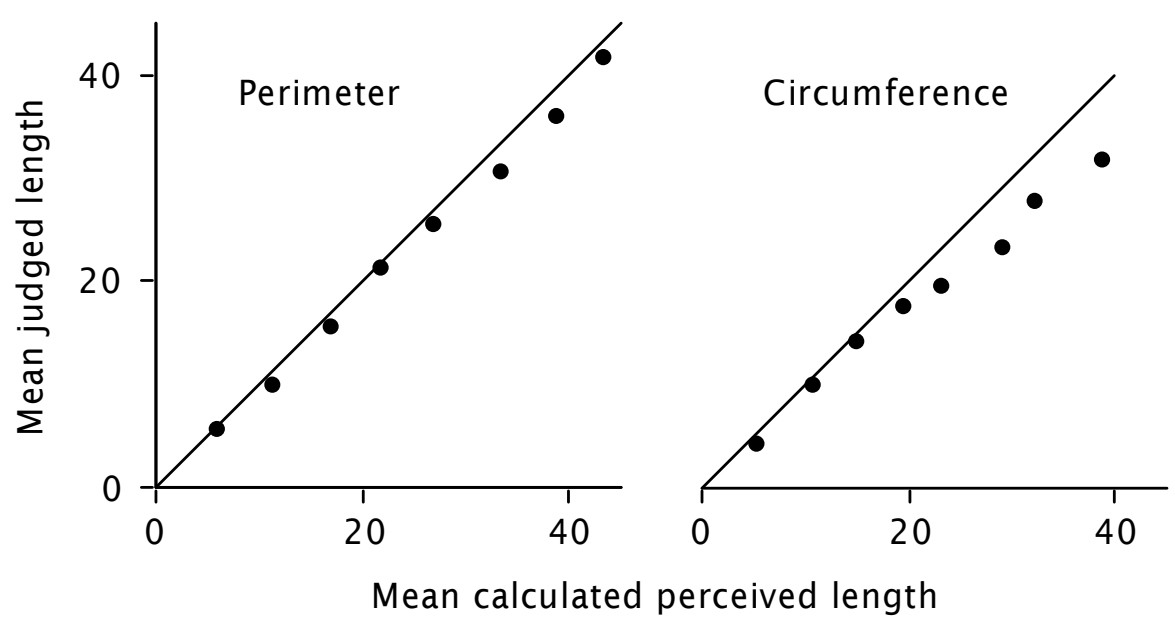

Figure 5. Mean judged lengths of perimeter and circumference plotted against the corresponding mean calculated perceived length

\subsection{Calculated Area}

Figure 6 shows mean judged square and disk areas plotted against mean $J_{L}^{2}$ and mean $\pi \cdot J_{L}^{2} / 4$, respectively. The left and right curves represent least-squares fits to a standard power function with exponent 0.99 and 1.03 , respectively. These exponents show that counts of mental units of length or area were fundamental measures.

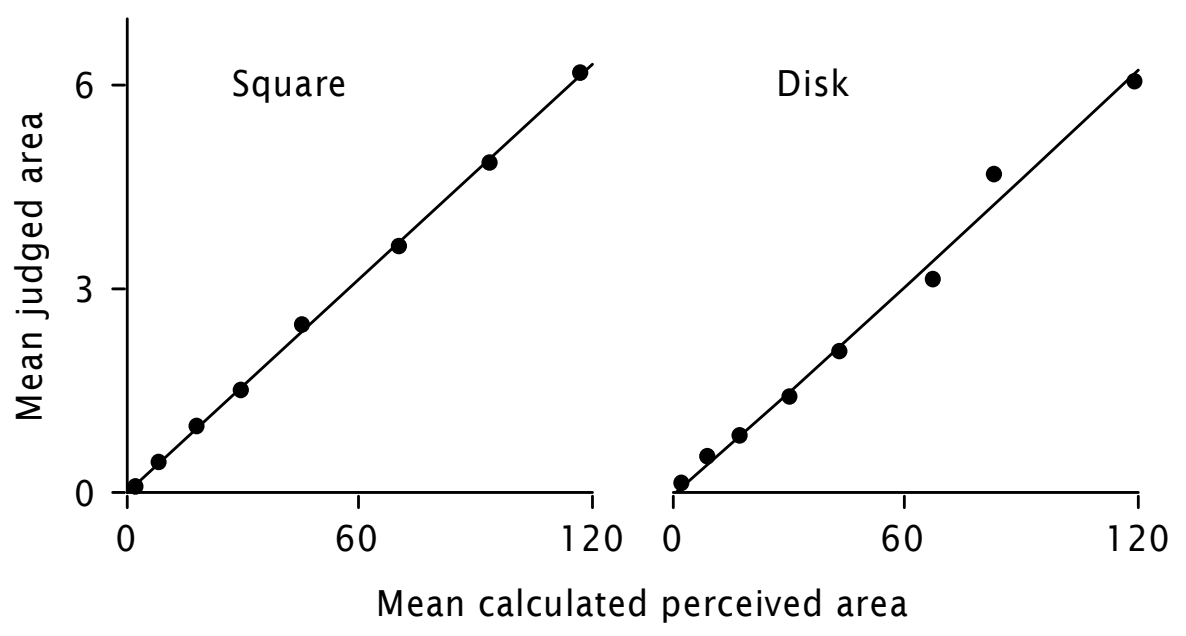

Figure 6. Mean judged areas of square and disk plotted against mean calculated perceived areas of square and disk, respectively 


\subsection{Introspective Reports}

\subsubsection{Length}

At the end of the experiment, all participants reported that they judged the perimeter by multiplying judged side by 4 . Eight participants reported judging the circumference by first imaginally straightening the circumference and subsequently counting the centimeters contained in it. Five reported straightening the entire circumference, one straightening half of it multiplying its judged length by 2 , and two straightening $1 / 4$ of it multiplying its judged length by 4 . The remaining participants reported concatenating one centimeter along the circumference without mentally straightening the circumference. Participants can compare distances along curves by mentally tracing the curves (Jolicoeur, Ullman, \& Mackay, 1991; Pringle \& Egeth, 1988). It is undecided whether the participants in the present study tried to verbalize mental tracing or a mental operation specific to the judgment task such as mental straightening.

\subsubsection{Area}

At the end of the experiment, nine and eight participants reported not knowing the formulas for calculating the circumference and area of a disk, respectively. Three participants recalled the formulas after a relatively long effort of memory. This effort and the related nonverbal behavior indicated that they did not recall these formulas during the experiment.

\section{General Discussion}

\subsection{Verification of Fundamental Measurement}

Concatenating a mental unit of measurement requires transformations of information: linear extents varying in inclination must be mentally rotated, curved extents must be mentally traced or straightened, and unit surfaces must be mentally transformed to be fit in the test surfaces. The present results show that mental transformations of information may impair the constancy of mental units during concatenation depending on extent length and surface shape.

If judgments of hypotenuse length are fundamental measures, Equation 1 predicts that the straight line with slope 1 and intercept 0 describes the relation between these judgments and perceived hypotenuse length calculated from judgments of leg length. In Figure 2 the left diagram shows that this prediction was closely confirmed only for relatively short hypotenuse lengths while the right diagram shows that this prediction was closely confirmed for all lengths of the hypotenuse when the nonconstancy of the mental unit for the hypotenuse was compensated by transforming $J_{H}$ to $J_{H}{ }^{1.023}$.

If judgments of leg length and area are fundamental measures, Equation 2 predicts that judgments of area are related linearly to perceived area calculated from corresponding judgments of leg length. In Figure 3 the left diagram shows that this prediction was closely confirmed when mental shape transformations were difficult. When this difficulty was minimized, Figure 6 shows that the prediction was more satisfactorily verified.

The results allow one to conclude that judgments of frontal length and frontal area made by mental counting are fundamental measures, at least approximately.

\subsection{Comparison of Methods}

\subsubsection{Magnitude Estimation and Rating}

Counting of units, magnitude estimation, and rating are distinct methods. For area, they yield exponents of the psychophysical power function of about 1, 0.8 , and 0.4 as shown in Figure 4, Wagner (2006, p. 87), and Stevens \& Guirao (1963), respectively. These differences in exponent may be interpreted as follows.

Complexity of numerical processing affects judgments (Baird, Kreindler, \& Jones, 1971; Barth \& Paladino, 2011; Booth \& Siegler, 2006; DeCarlo, 2005; Duda, 1975; Ekman, Hosman, Lindman, Ljungberg, \& Åkesson, 1968; Jones \& Marcus, 1961). Mental counting required minimal numerical processing. Magnitude estimation required at least multiplying counts of units by the modulus of the standard, calculating the reciprocal of these counts for test stimuli smaller than the standard, and processing the numerical information in the introductory example to the method. Rating required a more complicated numerical processing. Magnitude estimation and rating could have also involved the evaluation of quantity relations not required in the counting of mental units.

\subsubsection{Nonmetric Scaling}

The exponent of the psychophysical power function found by fundamental measurement is about 1 for length and area (Figures 1 and 4 ) and that found by nonmetric scaling varies from 0.46 to 0.87 for length and from 0.65 to 0.89 for area (Markley, Ayers, \& Rule, 1969; Parker, Schneider, \& Kanow, 1975; Petrusic, Baranski, \& 
Kennedy, 1998; Petrusic \& Jamieson, 1979; Rule \& Curtis, 1970; Schneider \& Bissett, 1988). Using nonmetric scaling, Young (1970) found an exponent of 1 for length. However, this result is not pertinent to our case since stimulus lines were varied in a very small range and were presented in conditions such that their lengths were barely discriminable. Using largely different stimuli, Schneider and Bissett (1988) found an exponent of 1 for both length and area in one group of participants. However, using the same stimuli, they found an exponent of 0.85 for length and of 0.89 for area in a different group of participants.

One can interpret these differences in exponent as follows. Assume the psychophysical function is

$$
\Psi=a \cdot \Phi^{\beta}+b
$$

with $a, b$, and $\beta$ constant and $\Psi$ and $\Phi$ the sensory and stimulus magnitudes, respectively. For $n$ values of $\Phi$ yielding $n$ different values of $\Psi$, for each possible $\beta$ there are $n \cdot(n-1) / 2$ absolute differences between values of $\Psi$. For each $\beta$, these differences may be ranked in terms of size, say, from the smallest to the largest. This ranking is independent of $a$ and $b$. We may call it the ranking of differences for short.

For a given $n$, nonmetric scaling requires each participant to generate a ranking of differences by subjectively ranking all possible absolute differences between values of $\Psi$. This empirical ranking of sensory differences is used to numerically recover an estimate of the corresponding $\beta$, assuming the response used by participants to rank the sensory differences varies monotonically with these differences (Shepard, 1962).

Equation 3 implies that there are ranges of values of $\beta$ within each of which each $\beta$ has the same ranking of differences. Numerically, with $\beta$ varying in steps of .01 and for the case of equidistant stimuli represented by the integers 1 to $n$ with $n=6,8,10$, or 20 as values for $\Phi$, I determined these ranges for all combinations of $\beta$ and $n$.

Figure 7 shows the results for each $n$. In each diagram the abscissa represents $\beta$ and the ordinate represents the ends of the range of the values of $\beta$ that specify the same ranking of differences specified by the corresponding $\beta$ represented on the abscissa. Thick and thin lines show the lower and upper ends of this range, respectively.

Figure 7 shows that the number of rankings of differences discriminated by nonmetric scaling increases with $n$. For $n=6$, this method discriminates between only four rankings of differences. Discrimination of rankings of differences may be barely acceptable for $n=8$ and may be satisfactory for $n=10$ or larger. These conditions of acceptability match those determined by numerical correlation analysis (Shepard, 1966).

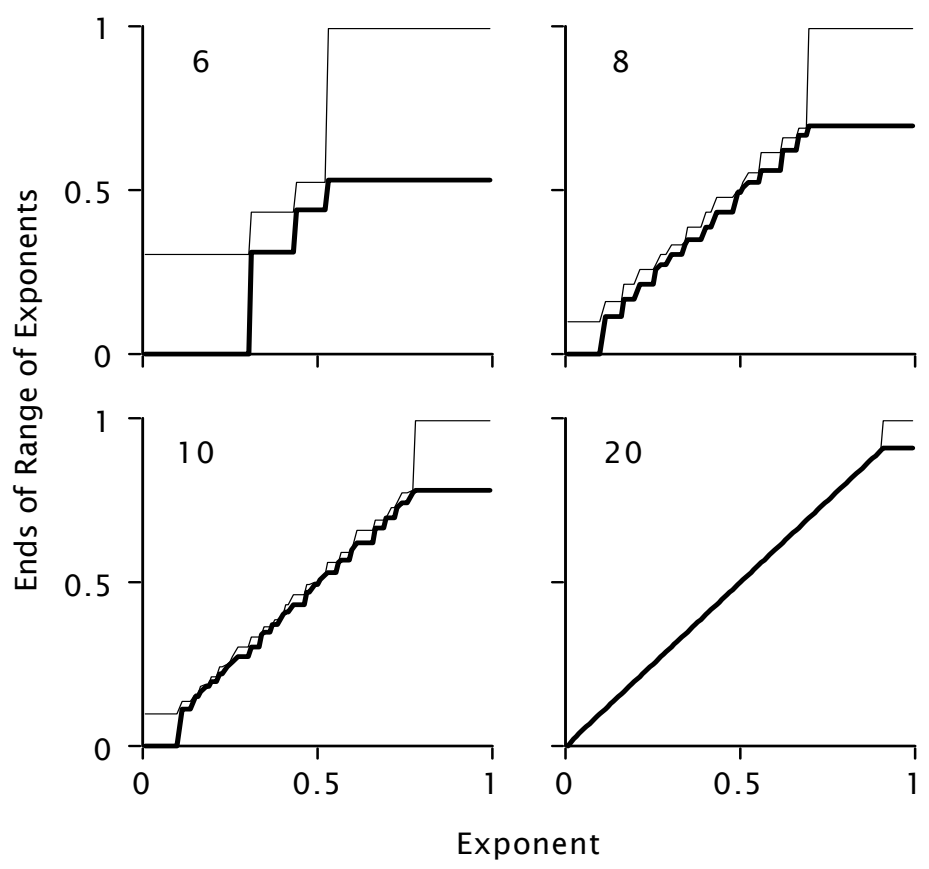

Figure 7. For $6,8,10$, or 20 stimuli, the abscissa represents the exponent of the psychophysical power function and the ordinate the ends of the range of the exponents that specify the same ranking of differences specified by the exponent on the abscissa. Thick and thin lines show the lower and upper ends of this range, respectively 
Nonmetric scaling may spuriously produces exponents lower than 1 for the following reason. Assume that $\beta=1$. Figure 7 shows that the ranking of differences specified by a potential $\beta$ of 1 is also specified by a large range of other values of $\beta$. This range is $0.53-1$ for $n=6,0.7-1$ for $n=8$, and $0.78-1$ for $n=10$. Thus, depending on the chosen $n$ from 6 to 10, nonmetric scaling may yield exponents ranging anywhere from 0.53 to 1 . The nonmetric scaling studies mentioned above used values of $n$ varying from 6 to 10 .

\section{Acknowledgements}

I wish to thank Norman H. Anderson, Lawrence E. Marks, Bruce Schneider, Robert Teghtsoonian, David J. Weiss, and Verena Zudini for providing useful feedback on an earlier version of this paper.

\section{References}

Anderson, N. H., \& Weiss, D. J. (1971). Test of a multiplying model for estimated area of rectangles. American Journal of Psychology, 84, 543-548. http://dx.doi.org/10.2307/1421171

Baird, J. C., Kreindler, M., \& Jones, K. (1971). Generation of multiple ratio scales with a fixed stimulus attribute. Perception \& Psychophysics, 9, 399-403. http://dx.doi.org/10.3758/BF03210237

Barth, H. C., \& Paladino, A. M. (2011). The development of numerical estimation: Evidence against a representational shift. Developmental Science, 14, 125-135. http://dx.doi.org/10.1111/j.1467-7687.2010.00962.x

Booth, J. L., \& Siegler, R. S. (2006). Developmental and individual differences in pure numerical estimation. Developmental Psychology, 42, 189-201. http://dx.doi.org/10.1037/0012-1649.41.6.189

Campbell, N. R. (1920). Physics: The elements. Cambridge: Cambridge University Press.

Cliff, N. (1992). Abstract measurement theory and the revolution that never happened. Psychological Science, 3 , 186-190. http://dx.doi.org/10.1111/j.1467-9280.1992.tb00024.x

DeCarlo, L. T. (2005). On bias in magnitude scaling and some conjectures of Stevens. Attention, Perception, \& Psychophysics, 67, 886-896. http://dx.doi.org/10.3758/BF03193541

Duda, P. D. (1975). Tests of the psychological meaning of the power law. Journal of Experimental Psychology: Human Perception and Performance, 1, 188-194. http://dx.doi.org/10.1037/0096-1523.1.2.188

Ekman, G., Hosman, B., Lindman, R., Ljungberg, L., \& Åkesson, C. Å. (1968). Interindividual differences in scaling performance. Perceptual \& Motor Skills, 26, 815-823. http://dx.doi.org/10.2466/pms.1968.26.3.815

Estes, W. K. (1975). Some targets for mathematical psychology. Journal of Mathematical Psychology, 12, 263-282. http://dx.doi.org/10.1016/0022-2496(75)90025-5

Falmagne, J. C. (1985). Elements of psychophysical theory. New York: Oxford University Press.

Giaquinto, M. (2007). Visual thinking in mathematics: An epistemological study. Oxford: Oxford University Press.

Guilford, J. P. (1954). Psychometric methods. New York: McGraw-Hill.

Hartley, A. A. (1977). Mental measurement in the magnitude estimation of length. Journal of Experimental Psychology: Human Perception and Performance, 3, 622-628. http://dx.doi.org/10.1037/0096-1523.3.4.622

Hartley, A. A. (1981). Mental measurement of line length: The role of the standard. Journal of Experimental Psychology: Human Perception and Performance, 7, 309-317. http://dx.doi.org/10.1037/0096-1523.7.2.309

Hölder, O. (1901). Die Axiome der Quantität und die Lehre vom Maß. Berichte über die Verhandlungen der Königlich Sächsischen Gesellschaft der Wissenschaften zu Leipzig, Mathematisch-Physische Klasse, 53, 1-64.

Jolicoeur, P., Ullman, S., \& Mackay, M. (1991). Visual curve tracing properties. Journal of Experimental Psychology: Human Perception and Performance, 17, 997-1022. http://dx.doi.org/10.1037/0096-1523.17.4.997

Jones, N., \& Marcus, M. J. (1961). The subject effect in judgments of subjective magnitude. Journal of Experimental Psychology, 61, 40-44. http://dx.doi.org/10.1037/h0046074

Krantz, D. H. (1972). A theory of magnitude estimation and cross-modality matching. Journal of Mathematical Psychology, 9, 168-199. http://dx.doi.org/10.1016/0022-2496(72)90025-9

Markley, R. P., Ayers, D., \& Rule, S. J. (1969). Similarity judgments of line length. Perception \& Psychophysics, 6, 58-60. http://dx.doi.org/10.3758/BF03210678

Masin, S. C., \& Vidotto, G. (1983). A magnitude estimation study of the inverted-T illusion. Perception \& Psychophysics, 33, 582-584. http://dx.doi.org/10.3758/BF03202941 
Michell, J. (2003). Epistemology of measurement: The relevance of its history for quantification in the social sciences. Social Science Information, 42, 515-534. http://dx.doi.org/10.1177/0539018403424004

Parker, S., Schneider, B., \& Kanow, G. (1975). Ratio scale measurement of the perceived lengths of lines. Journal of Experimental Psychology: Human Perception and Performance, 1, 195-204. http://dx.doi.org/10.1037/0096-1523.1.2.195

Petrusic, W. M., Baranski, J. V., \& Kennedy, R. (1998). Similarity comparisons with remembered and perceived magnitudes: Memory psychophysics and fundamental measurement. Memory \& Cognition, 26, 1041-1055. http://dx.doi.org/10.3758/BF03201182

Petrusic, W. M., \& Jamieson, D. G. (1979). Resolution time and the coding of arithmetic relations on supraliminally different visual extents. Journal of Mathematical Psychology, 19, 89-107. http://dx.doi.org/10.1016/0022-2496(79)90014-2

Pringle, R., \& Egeth, H. E. (1988). Mental curve tracing with elementary stimuli. Journal of Experimental Psychology: Human Perception and Performance, 14, 716-728. http://dx.doi.org/10.1037/0096-1523.14.4.716

Reed, S. K., Hock, H. S., \& Lockhead, G. (1983). Tacit knowledge and the effect of pattern recognition on mental scanning. Memory and Cognition, 11, 137-143. http://dx.doi.org/10.3758/BF03213468

Reese, T. V. (1943). The application of the theory of physical measurement to the measurement of psychological magnitudes, with three experimental examples. Psychological Monographs, 55(3), 1-89. http://dx.doi.org/10.1037/h0093539

Rentmeister-Bryant, H., Slotnick, B., \& Parker, S. (2000). Subillusions in the horizontal-vertical illusion. In C. Bonnet (Ed.), Proceedings of the sixteenth annual meeting of the International Society for Psychophysics (pp. 291-295). Strasbourg, France: International Society for Psychophysics.

Rule, S. J., \& Curtis, D. W. (1970). Input and output transformations from magnitude estimation. Journal of Experimental Psychology, 86, 343-349. http://dx.doi.org/10.1037/h0030154

Schneider, B., \& Bissett, R. (1988). "Ratio" and "difference" judgments for length, area, and volume: Are there two classes of sensory continua? Journal of Experimental Psychology: Human Perception and Performance, 14, 503-512. http://dx.doi.org/10.1037/0096-1523.14.3.503

Schönemann, P. H. (1994). Measurement: The reasonable ineffectiveness of mathematics in the social sciences. In I. Borg \& P. Ph. Mohler (Eds.), Trends and perspectives in empirical social research (pp. 149-160). Berlin: de Gruyter.

Shepard, R. N. (1962). The analysis of proximities: Multidimensional scaling with an unknown distance function. I. Psychometrika, 27, 125-140. http://hdl.handle.net/10.1007/BF02289630

Shepard, R. N. (1966). Metric structures in ordinal data. Journal of Mathematical Psychology, 3, 287-315. http://dx.doi.org/10.1016/0022-2496(66)90017-4

Stevens, S. S., \& Galanter, E. H. (1957). Ratio scales and category scales for a dozen perceptual continua. Journal of Experimental Psychology, 54, 153-181. http://www.ncbi.nlm.nih.gov/pubmed/13984045

Stevens, S. S., \& Guirao, M. (1963). Subjective scaling of length and area and the matching of length to loudness and brightness. Journal of Experimental Psychology, 66, 177-186. http://dx.doi.org/10.1037/h0044984

Teghtsoonian, R., \& Teghtsoonian, M. (1971). The apparent length of perimeters and diameters define a ratio smaller than pi. American Journal of Psychology, 84, 437-438. http://www.jstor.org/stable/1420474

Uhlarik, J., Pringle, R., Jordan, K., \& Misceo, G. (1980). Size scaling in two-dimensional pictorial arrays. Perception \& Psychophysics, 27, 60-70. http://dx.doi.org/10.3758/BF03199907

Wagner, M. (2006). The geometries of visual space. Mahwah, NJ: Erlbaum.

Weiss, D. J., \& Gardner, G. S. (1979). Subjective hypotenuse estimation: A test of the Pythagorean theorem. Perceptual and Motor Skills, 48, 607-615. http://dx.doi.org/10.2466/pms.1979.48.2.607

Young, F. W. (1970). Nonmetric scaling of line lengths using latencies, similarity, and same-different judgments. Perception \& Psychophysics, 8, 363-369. http://dx.doi.org/10.3758/BF03212609

Zudini, V. (2011). The Euclidean model of measurement in Fechner's psychophysics. Journal of the History of the Behavioral Sciences, 47, 70-87. http://dx.doi.org/10.1002/jhbs.20472 